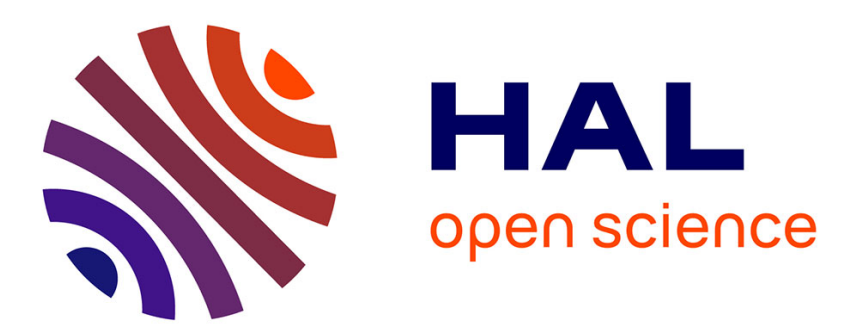

\title{
Stability Analysis of an Array of Magnets: When Will It Jump?
}

Nicolas Taberlet, Jérémy Ferrand, Nicolas Plihon

\section{To cite this version:}

Nicolas Taberlet, Jérémy Ferrand, Nicolas Plihon. Stability Analysis of an Array of Magnets: When Will It Jump?. Physical Review Letters, 2018, 120 (264301), 10.1103/PhysRevLett.120.264301 . hal02399069

\section{HAL Id: hal-02399069 https://hal.science/hal-02399069}

Submitted on 8 Dec 2019

HAL is a multi-disciplinary open access archive for the deposit and dissemination of scientific research documents, whether they are published or not. The documents may come from teaching and research institutions in France or abroad, or from public or private research centers.
L'archive ouverte pluridisciplinaire HAL, est destinée au dépôt et à la diffusion de documents scientifiques de niveau recherche, publiés ou non, émanant des établissements d'enseignement et de recherche français ou étrangers, des laboratoires publics ou privés. 


\title{
Stability Analysis of an Array of Magnets: When Will It Jump?
}

\author{
Nicolas Taberlet, ${ }^{*}$ Jérémy Ferrand, and Nicolas Plihon \\ Univ Lyon, Ens de Lyon, Univ Claude Bernard Lyon 1, CNRS, Laboratoire de Physique, F-69342 Lyon, France
}

(Received 7 March 2018; published 29 June 2018)

\begin{abstract}
A bidimensional array of magnets whose magnetic moments share the same vertical orientation, and lying on a planar surface, can be gradually compacted. As the density reaches a threshold, the assembly becomes unstable, and the magnets violently pop out of plane. In this Letter, we investigate experimentally and theoretically the maximum packing fraction (or density) of a bidimensional planar assembly of identical cylindrical magnets. We show that the instability can be attributed to local fluctuations of the altitude of the magnets on the planar surface. The maximum density is theoretically predicted assuming dipolar interactions between the magnets and is in excellent agreement with experimental results using a variety of cylindrical magnets.
\end{abstract}

DOI: 10.1103/PhysRevLett.120.264301

The remarkable behavior of an assembly of magnets has drawn interest for more than a century. Ewing proposed an analog experiment with compass needles in a fixed 2D array to study hysteresis cycles of the magnetic properties of crystals as early as 1890 [1]. These experiments have recently been revisited in order to study the thermodynamics of dipolar lattices [2] or frustrated magnetic systems at the macroscopic scale [3] or at the nanoscale [4-6]. Nonlinear waves encountered in solids, ionic crystals [7], or plasma crystals [8] have also been widely studied in analog experiments employing magnets free to move along one direction, showing behaviors ranging from dispersive waves [9] to moving breathers [10] or solitary waves [11]. The behavior of turbulent dynamos have also been reproduced using magnets assemblies [12], and the spontaneous dynamics of unconstrained chains of magnets have recently attracted attention [13-15].

In this Letter, we elucidate the physical mechanisms limiting the maximum packing fraction of a planar assembly of magnets whose magnetic moments are aligned in the same (vertical) direction. This study was motivated by the recently released game Magination [16] in which magnets are placed one by one on a supporting surface, and the resulting repulsive magnetic interactions cause the system to self-organize (Fig. 1 and Supplemental Material A [17]). As the density of magnets increases, the horizontal forces acting between them increase, but the magnets manage to stay in contact with the supporting surface. However, when the density exceeds a critical value, the assembly becomes unstable (see the videos in the Supplemental Material [17] and Fig. 1). This Letter reports experimental and theoretical findings on this threshold.

Two effects can trigger this sudden transition: a particular magnet has to be submitted to either a net vertical magnetic force (which exceeds its weight) or to a horizontal magnetic torque (which exceeds the moment of its own weight). In an ideal situation where all magnets were perfectly aligned on an ideal plane [Fig. 2(a)], the symmetry of the geometry would forbid any net vertical magnetic force or any magnetic torque. However, any fluctuation in either the altitude of the magnets or in the nearly vertical orientation of their magnetic moments [Fig. 2(b)] could trigger a transition. Both sources of misalignment can be due to imperfections in the supporting surface, the magnets themselves, or due to dust particles, virtually invisible to the naked eye. In the remainder, the effect of both the force and the torque are investigated separately in order to elucidate if one prevails over the other.

Here, we investigate these effects through experiments performed using a variety of cylindrical magnets and derive theoretical predictions, in the small-perturbation limit, assuming a permanent dipole-dipole interaction between the magnets.

Experimental details.-The magnets are placed on a horizontal surface (a $5 \mathrm{~mm}$ thick PMMA plate), lubricated using a dry powder lubricant which yields a friction coefficient as low as $0.05(20 \mu \mathrm{m}$ glass beads, Potters industries [18]) which allows them to easily rearrange as the density is gradually increased. Experiments were performed using flat cylindrical magnets whose aspect ratio (height to diameter) ranges from 0.2 to 1 . We used grade $\mathrm{Y} 35$ ferrite $(\mathrm{Fe})$ or grade $\mathrm{N} 42 \mathrm{NdFeB}$ alloys $(\mathrm{Nd})$ magnets [19], whose diameters range from 5 to $30 \mathrm{~mm}$ and heights range from 3 to $10 \mathrm{~mm}$. As a convention, a $10 \mathrm{~mm}$ diameter and $3 \mathrm{~mm}$ high ferrite magnet will be labeled Fe-10-3. Note that for the densities we have studied, the magnetic field created by the nearest neighbors of a magnet largely exceed the ambient field, which will therefore be neglected in the remainder.

The orientations of the magnets' magnetic moments $\mu$ are to the first order identical and perpendicular to the plane 


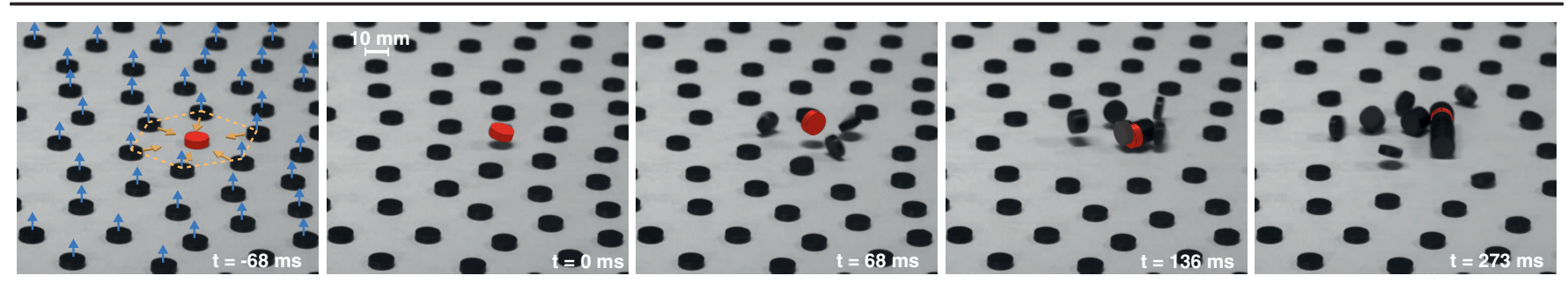

FIG. 1. Time sequence showing the dramatic out-of-plane burst of magnets assembly as the critical density is reached. The first magnet bursting at $t=0$ is highlighted in red. Magnetic dipoles $\mu$ appear as blue arrows, while the forces from the first neighbors of the hexagonal pattern are shown as orange arrows on the left.

(see the study of their variability in Supplemental Material B [17]), so that any two magnets exert a repulsive horizontal force on one another. The magnets are circumscribed within a given surface by confining walls against which the outermost magnets rest. The density of magnets can be increased either by adding magnets to a system of constant surface area or by bringing the bounding walls closer.

In an inhomogeneous packing, when the density is high enough, the net horizontal force exerted on one magnet can exceed the friction forces and cause the magnet to move towards a region of lower density. As the packing fraction is gradually increased, the system therefore rearranges and tends to be uniform. Note that in order to help this process, the plane can be cautiously horizontally shaken. Figure 1 shows that the magnet coming out of the plane is surrounded by six magnets in hexagon. We have verified (see Supplemental Material A [17]) that the magnets are placed on a hexagonal pattern [20] (except at the edges), and we assume that this is the case later on.

Modeling.-As mentioned above, the physical origin of the instability can be attributed to the existence of a net vertical magnetic force [if the altitude of a magnet is

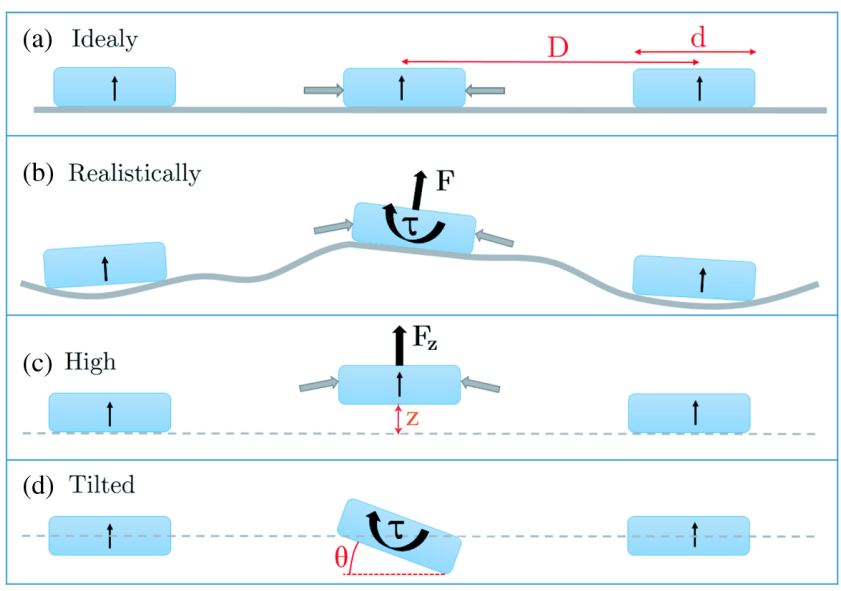

FIG. 2. Schematics of an ideally planar system (a) and realistic situation with slighted misaligned magnets (b). Model cases in which (c) only the altitude $z$ of the central magnet varies (creating a net vertical force $F_{z}$ ) and (d) in which the central magnet is inclined by $\theta$ (creating a torque $\tau$ ). positive, Fig. 2(c)] or to a magnetic torque [if a magnet is not ideally horizontal, Fig. 2(d)]. The stability of the system is therefore the result of two competing effects: the weight of the magnets (stabilizing) and the magnetic efforts (destabilizing) caused by the surrounding magnetic moments. Stability criteria are derived for magnets of mass $m$ and diameter $d$ and where $z$ is the altitude, in the case of Fig. 2(c), and $\theta$ its inclination (due to surface profile or inclination of magnetic moment from the ideal case), in the case of Fig. 2(d), and $g$ acceleration due to gravity. We checked the validity of the dipolar approximation on the spatial evolution of the magnetic fields as well as on the interactions between magnets (see the Supplemental Material C [17]). In order to conveniently compare the competing effects of gravity and magnetism, a characteristic length $L_{0}$ is introduced:

$$
L_{0}^{4} \equiv \frac{3 \mu_{0} \mu^{2}}{4 \pi m g}
$$

where $\mu_{0}$ is the magnetic permeability of vacuum and $\mu$ is the magnetic moment of the magnets. Note that not only is $L_{0}$ a simple way to normalize the distances but it also provides a useful method for measuring the moment of a magnet. A magnet is glued to a nearly vertical plane (its moment being horizontal and perpendicular to the plane). A second magnet resting on the same vertical plane (its moment being aligned with the first one) is allowed to slide along a vertical line above the first one. Here, $L_{0}$ is the equilibrium distance between the centers of the magnets for which the magnetic force acting on the top one counterbalances its weight $m g$. Thus, $L_{0}$ is a length which characterizes the competition between weight and magnetic forces.

Six magnetic moments $\mu$ aligned along the vertical direction located at the vertices of a planar hexagon of size $D$ (the smallest distance between lattice points) create a vertical magnetic field at its center $\mathbf{B}=6 \mu_{0} \mu /\left(4 \pi D^{3}\right) \mathbf{u}_{z}$. The net vertical force acting on a magnet placed at the center is given by $\mu \partial_{z} B_{z}$, while the torque it experiences is given by $\boldsymbol{\mu} \times \mathbf{B}$. Assuming a dipole-dipole interaction, one finds, in the limit of small altitude $z$ (see calculations in Supplemental Material C [17]): 

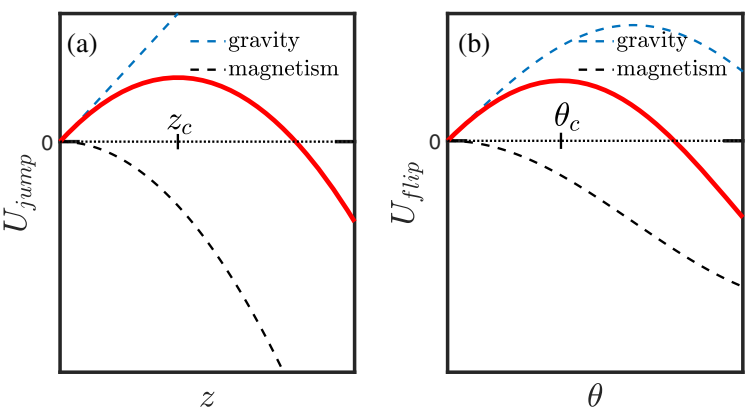

FIG. 3. Energy landscapes. (a) Corresponds to situations with $\theta=0$ and only the potential of vertical magnetic force and (b) to situations with $z=0$ with only the potential of magnetic torque. The red lines are the sum of gravitational and magnetic potential energies.

$$
F_{z}=6 \times 9 \frac{\mu_{0} \mu^{2}}{4 \pi} \frac{z}{D^{5}}, \quad \tau=6 \frac{\mu_{0} \mu^{2}}{4 \pi D^{3}} \sin \theta
$$

These conservative forces derive from potential energies, to which the gravitational potential energy can be compared: $U_{g}=m g z$ for the force, and $U_{g}=\frac{1}{2} m g d \sin \theta$ for the torque. Figure 3 sketches the corresponding energy landscapes for positive values. Both profiles show an energy well for low altitudes and inclinations. As a side note, one can realize that in this athermal system (the thermal energy $k_{B} T$ being exceedingly negligible), a stable configuration cannot overcome (for which one magnet would jump out of plane) the energy barriers. There is, however, a critical altitude $z_{c}$ and inclination $\theta_{c}$ above which the system becomes unstable. In the following, we examine which of the two effects is dominant.

The central magnet is unstable if the net magnetic force exceeds its weight (for critical altitude $z_{c}$ ) or if the torque exceeds that of its weight (for a critical inclination $\theta_{c}$ ):

$$
\frac{z_{c}}{L_{0}}=\frac{1}{18} \frac{D^{5}}{L_{0}^{5}}, \quad \tan \theta_{c}=\frac{d D^{3}}{4 L_{0}^{4}} .
$$

Experimental validation.-The maximum packing fraction was measured experimentally using two methods for Fe-10-3. In the first one, a magnet rests on the supporting surface, while six surrounding magnets are held at the vertices of a virtual hexagon using individual supports and are gradually brought closer. In this case, there is no need for a dry lubricant. The maximum density (or smallest distance $D$ ) is measured when the central magnet jumps out of the plane.

In the second method, six magnets are glued on the supporting surface (a $5 \mathrm{~mm}$ thick PMMA plate) and form a hexagon, which imposes a fixed density. A free magnet is placed at the center as the plate itself rests on a thick steel plane which holds the central magnet down and prevents it from jumping out of plane (again without dry lubricant). The metal plate is slowly lowered, and the packing is considered to be stable if the central magnet remains on the surface during the process. The first method is clearly easier to implement but was thought to be less accurate since the central magnet tends to move sideways during the compaction process. However, the maximum density measured from the two methods are identical (with uncertainties of less than $1 \mathrm{~mm}$ on the smallest distance $D$ ). For practical reasons, a simplified protocol, in which only three surrounding magnets (at the vertices of an equilateral triangle) are gradually brought closer, was used for the other set of magnets. Note that in this case the numerical prefactors in (3) need to multiplied by 2 . Table I summarizes the experimental results for $L_{0}$ and $D$ in the case of triangle configuration and the corresponding values of $z_{c}$ and $\theta_{c}$ computed using (3) (with numerical prefactors multiplied by 2 ).

The direct interpretation of Table I is not straightforward, though the critical inclinations for ferrite magnets are rather high; indeed, a $5.7^{\circ}$ inclination of a $15 \mathrm{~mm}$ in diameter magnet would require one side to be $1.5 \mathrm{~mm}$ higher than the other. Such a tilt angle would be very obvious to the naked eye and clearly unrealistic for magnets resting on a PMMA plane. Note that the fluctuations of the orientation of the magnetic moment with respect to the body of the magnets remain below $0.6^{\circ}$ (as shown in Supplemental Material B [17]), which is considerably smaller than the $\theta_{c}$ values reported in Table I. Our experimental results may be recast assuming typical values for the surface flatness and inclination. The typical elevation is linked to the surface flatness of a standard PMMA plate, much larger than the typical $0.1 \mathrm{~mm}$ surface flatness of a research-grade optical table [21]. However, in addition to the impurities present on the surface, fine particles of metal often remain stuck to magnets, and we estimate the typical elevation as being $1 \mathrm{~mm}$. Thus, we estimate the altitude differences between 100 microns and $1 \mathrm{~mm}$, which are much larger than the typical size of the dry lubricant powder. As for the angle, we hypothesize that it is only due to the topography of the surface. Thus, the extreme angles are calculated simply by

TABLE I. Minimum distance $D$, characteristic distance $L_{0}$, corresponding critical altitude $z_{c}$, and inclination $\theta_{c}$ for a variety of magnets. Incertitudes for $z_{c}$ and $\theta_{c}$ come from measurements of $D$ and $L_{0}$ not shown in the table.

\begin{tabular}{lcccc}
\hline \hline Type & $D(\mathrm{~mm})$ & $L_{0}(\mathrm{~mm})$ & $z_{c}(\mathrm{~mm})$ & $\theta_{c}(\mathrm{deg})$ \\
\hline Fe-5-5 & 14.4 & 17 & $0.67 \pm 0.15$ & $4.6 \pm 0.6$ \\
Fe-10-3 & 17.1 & 21 & $0.84 \pm 0.24$ & $7.4 \pm 1.2$ \\
Fe-15-3 & 20.8 & 28 & $0.59 \pm 0.07$ & $5.7 \pm 0.4$ \\
Fe-25-10 & 31.7 & 45 & $0.73 \pm 0.21$ & $5.0 \pm 0.8$ \\
Nd-10-3 & 23.1 & 32 & $0.50 \pm 0.13$ & $2.7 \pm 0.4$ \\
Nd-10-5 & 23.8 & 37 & $0.34 \pm 0.14$ & $1.7 \pm 0.4$ \\
Nd-20-10 & 38.7 & 63 & $0.46 \pm 0.13$ & $1.7 \pm 0.3$ \\
Nd-30-6 & 47.9 & 78 & $0.45 \pm 0.35$ & $1.9 \pm 0.8$ \\
\hline \hline
\end{tabular}



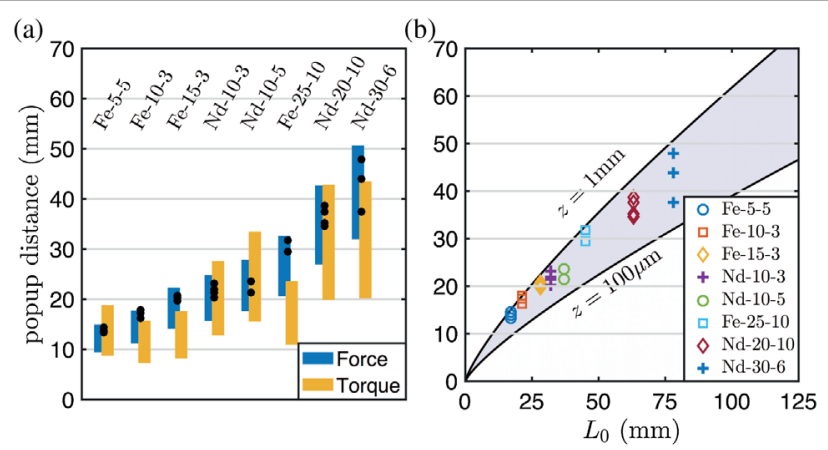

FIG. 4. Popup distance measured experimentally and predicted range $\left\{\right.$ see (3) with $\left.z_{c}=[0.1-1] \mathrm{mm}\right\}$. (a) Minimum distance $D$ for a variety of magnets. Lines: for vertical force (left, blue) and torque (right, yellow) Black points: experimental values. (b) Same experimental points plotted as function of distance $L_{0}$. Limits are only for vertical force.

making the ratio between the previous heights and the diameter of the magnet. From these extrema, the corresponding distances $D$ (i.e., the maximum density) may be computed from (3) for the various values of $L_{0}$. Figure 4(a) shows the experimental data of the popup distance $D$ (black dots), along with the predicted values of $D$ due to vertical force (left, blue) and torque (right, yellow). It can be seen that all experimental points are within the ranges of theoretically predicted distances for force or torque or both. For some magnets (Nd-30-6, Fe-25-10, Fe-15-3, $\mathrm{Fe}-10-3)$, we can state that the vertical force is responsible for the jump of the magnet. For all other magnets used, it is impossible to identify whether only the force or torque is responsible for the take-off of the magnet: both effects must be taken into account. Intuitively, the narrower and higher the magnet is, the more likely it will tip over and not take off with vertical force. The length $L_{0}$ and therefore the magnetic moment of the magnet $\mu$ has a great importance in the problem. We present only results for magnets with an aspect ratio lower than 1 . Indeed, for magnets with an aspect ratio larger than 1 , the assembly is highly unstable. Note that the demagnetization of the magnets is a secondorder effect as discussed in Supplemental Material D [17].

In order to visualize more clearly the limits for surface defects, Fig. 4(b) shows the minimum popup distance for vertical force as a function of the length $L_{0}$ characterizing the magnet. We see, as shown in Fig. 4(a) that all experimental points lie between the two critical height limits $z_{c}=100 \mu \mathrm{m}$ or $1 \mathrm{~mm}$. This graph makes it possible to draw two conclusions: it is not necessary to take the torque into account (with the magnets used here) to explain the take-off of the magnet, but the values in Fig. 4(a) seem to affirm that both effects are important in some cases. In addition, all experimental points are closer to the $1 \mathrm{~mm}$ limit than the 100 micron limit. This may have two explanations: either our surfaces are not smooth enough and clean enough to reach 100 microns of defect size, or the two effects (strength and torque) complement each other to further destabilize the system.

Applicability to the initial protocol.- - It should be noted that the data presented in Table I and Fig. 4 were obtained with a single free magnet at the center of a ring of fixed magnets. In the case of a large array (as Fig. 1), not all magnets can statistically stay at the lower limit. Instead, as the number of magnets increases, it becomes more and more probable than one reaching the upper limit. The system being as unstable as its weakest link (the most unstable magnet), the maximum density of a large assembly can therefore be determined using the upper limit $\left(z_{c} \simeq 1 \mathrm{~mm}\right)$. Note also that (3) was derived by taking into account only the six closest neighboring magnets. However, with the rapid decrease of the magnetic field with distance, this provides an excellent first order approximation. A complete numerical computation indeed shows that taking an infinite number of magnets into account would only reduce the critical distance $D$ by less than $3 \%$.

For the Fe-10-3 magnets shown in Fig. 1, the predicted value of the critical distance is $D=17.2 \mathrm{~mm}$, which corresponds to a density of $n=2 /\left(\sqrt{3} D^{2}\right) \simeq 39$ magnets $/ \mathrm{dm}^{2}$. However, using the compaction protocol presented in Fig. 1, the maximum density reached before the system blasts never exceeds 16 magnets $/ \mathrm{dm}^{2}(D=27 \mathrm{~mm})$, less than half of the predicted value. The protocol used can explain this discrepancy.

It is indeed virtually impossible for the system to homogeneously rearrange as the density is gradually increased. Even if all four confining walls were simultaneously and steadily brought closer, the density would always be greater near the edges. When one wall is moved, compaction (i.e., density) waves propagate throughout in the system, creating strong local fluctuations in the density. Moreover, the system can be trapped in a frustrated state, from which an increase in density would require global rearrangements rather than local adjustments.

The maximum density may also be measured using a protocol similar to the second method presented above. The outermost magnets of a hexagonal pattern (of predefined density) are glued to the PMMA plate so as to form a rigid confining boundary. A test magnet is then set at the center of the pattern as the plate rests on a steel plane, and the stability of the pattern is probed when lifting the PMMA plate away from the steel plane. Note that a compromise has to be found since thin PMMA plates can easily bend (as it is lifted from the metal plate), while thicker plates would reduce the attractive force exerted by the steel on the magnets. We were able to produce stable arrays of 61 Fe-10-3 magnets (i.e., a hexagonal patter of 9 units diameter) with a lattice $D=19 \mathrm{~mm}$, while arrays of pitch $D=17 \mathrm{~mm}$ and $D=18 \mathrm{~mm}$ never managed to remain stable upon removal of the metal plate. This second protocol therefore allows one to achieve densities that are very close to the theoretical prediction $(D=17.2 \mathrm{~mm})$. 
Conclusion.-The physical mechanisms which limit the maximum density of an array of identical magnets were elucidated. Our experimental results and theoretical predictions (based on dipolar interactions) indicate that the critical density above which the system becomes unstable results from a competition between the magnetic forces and torques on the one hand (due to irregularities of the surface and/or the magnets themselves) and gravity on the other hand, the ratio of these effects being characterized by the length $L_{0}$. While the predicted maximum density can be difficult to reach by individually adding magnets in a given perimeter or by gradually compressing an array, we propose a protocol in which the density is set to a chosen value while the magnets are held down onto the plane. By gradually relieving this constraint, one is able to obtain stable assemblies of magnets with a density very close to the theoretical limit.

Our experiments and theoretical predictions were performed for a hexagonal pattern of magnets (the natural configuration), but it would certainly be interesting to study the effect of defects in the crystalline order or to extend our work to other crystalline arrangements, disordered structures, or polydisperse assemblies of magnets. Moreover, our stability analysis shows that the overall stability of the system is determined by that of its most unstable magnet (or weakest link), but the collective dynamics of the collapse of the entire array once a single magnet has popped out of plane seems to be very rich and should deserve further investigation.

The authors acknowledge support from the PALSE program of the University of Lyon, the University Lyon Claude Bernard, the Société Française de Physique, Centre national de la recherche scientifique (CNRS), and from the École Normale Supérieure de Lyon and its Physics Department and Laboratoire de Physique.

*nicolas.taberlet@ens-lyon.fr

[1] J.A. Ewing, Contributions to the molecular theory of induced magnetism, Philos. Mag., 30, 205 (1890).

[2] E. Olive and P. Molho, Thermodynamic study of a lattice of compass needles in dipolar interaction, Phys. Rev. B 58, 9238 (1998).

[3] P. Mellado, A. Concha, and L. Mahadevan, Macroscopic Magnetic Frustration, Phys. Rev. Lett. 109, 257203 (2012).

[4] R. F. Wang, C. Nisoli, R. S. Freitas, J. Li, W. McConville, B. J. Cooley, M. S. Lund, N. Samarth, C. Leighton, V. H. Crespi, and P. Schiffer, Artificial spin ice in a geometrically frustrated lattice of nanoscale ferromagnetic islands, Nature (London) 439, 303 (2006).

[5] S. Zhang, J. Li, I. Gilbert, J. Bartell, M. J. Erickson, Y. Pan, P. E. Lammert, C. Nisoli, K. K. Kohli, R. Misra,
V. H. Crespi, N. Samarth, C. Leighton, and P. Schiffer, Perpendicular Magnetization and Generic Realization of the Ising Model in Artificial Spin Ice, Phys. Rev. Lett. 109, 087201 (2012).

[6] A. Farhan, P. M. Derlet, A. Kleibert, A. Balan, R. V. Chopdekar, M. Wyss, J. Perron, A. Scholl, F. Nolting, and L. J. Heyderman, Direct Observation of Thermal Relaxation in Artificial Spin Ice, Phys. Rev. Lett. 111, 057204 (2013).

[7] M. G. Raizen, J. M. Gilligan, J. C. Bergquist, W. M. Itano, and D. J. Wineland, Ionic crystals in a linear Paul trap, Phys. Rev. A 45, 6493 (1992).

[8] V. Nosenko, K. Avinash, J. Goree, and B. Liu, Nonlinear Interaction of Compressional Waves in a 2D Dusty Plasma Crystal, Phys. Rev. Lett. 92, 085001 (2004).

[9] A. Mehrem, N. Jiménez, L. J. Salmerón-Contreras, X. García-Andrés, L. M. García-Raffi, R. Picó, and V. J. Sánchez-Morcillo, Nonlinear dispersive waves in repulsive lattices, Phys. Rev. E 96, 012208 (2017).

[10] F. M. Russell, Y. Zolotaryuk, J. C. Eilbeck, and T. Dauxois, Moving breathers in a chain of magnetic pendulums, Phys. Rev. B 55, 6304 (1997).

[11] M. Moleron, A. Leonard, and C. Daraio, Solitary waves in a chain of repelling magnets, J. Appl. Phys. 115, 184901 (2014).

[12] N. Plihon, S. Miralles, M. Bourgoin, and J.-F. Pinton, Stochastic reversal dynamics of two interacting magnetic dipoles: A simple model experiment, Phys. Rev. E 94, 012224 (2016).

[13] J. Boisson, C. Rouby, J. Lee, and O. Doaré, Dynamics of a chain of permanent magnets, Europhys. Lett., 109, 34002 (2015).

[14] N. Vandewalle and S. Dorbolo, Magnetic ghosts and monopoles, New J. Phys., 16, 013050 (2014).

[15] B. A. Grzybowski, H. A. Stone, and G. M. Whitesides, Dynamic self-assembly of magnetized, millimetre-sized objects rotating at a liquid-air interface, Nature (London) 405, 1033 (2000).

[16] Magination Game, see the various videos at https:// maginationgame.com/.

[17] See Supplemental Material at http://link.aps.org/ supplemental/10.1103/PhysRevLett.120.264301 for the verification that the magnet network obtained is indeed hexagonal, the measurements of fluctuations in the orientation and amplitude of the magnetic moment of the magnets, the measurement of the field of the magnets and all interactions between two magnets (force, torque). Finally we discuss the demagnetization of the magnets. Two videos show the phenomenon in addition to Fig. 1 of the Letter.

[18] Glass beads, https://www.pqcorp.com/brands/pottersindustries.

[19] Supermagnete, https://www.supermagnete.com/.

[20] Q. Du, V. Faber, and M. Gunzburger, Centroidal Voronoi tessellations: Applications and algorithms, SIAM Rev., 41, 637 (1999).

[21] Thorlabs Optical Tables, https://www.thorlabs.com/ navigation.cfm?guide_id=41. 